\title{
Effect of Scattering Parameters On MIMO System Capacity
}

\author{
M. Tausif Afzal Rana1, M. Arif Khan2 , Waseem Afzal3,M. Zeeshan Baig3 and \\ Danish Akhtar1 \\ 1Electrical Engineering, ISRA University, Islamabad Campus, 44000, Pakistan \\ 2School of Computing and Mathematics, Charles Sturt University, Wagga 2650, Australia \\ 3Electronic Engineering, Mohammad Ali Jinnah University, Islamabad, 44000, Pakistan
}

\begin{abstract}
In this paper, the influence of scattering parameters on MIMO system capacity are discussed by designing MIMO antennas. In principle, the capacity of MIMO system can be increased by installing a high number of antennas both at the transmitter and receiver. However, scattering parameters also play a vital role in the capacity of MIMO system. This behavior of capacity is observed in case of partially and fully correlated environment in the presence of scattering parameters.
\end{abstract}

Keywords: MIMO, Capacity, Scattering Matrix, Antenna Array and Ansoft HFSS.

\section{Introduction}

In order to fulfil the requirement of growing technology, high data rate play a key role in wireless communication. The most promising of such a technology is Multiple Input Multiple Output, (MIMO) base antenna systems. MIMO system consist of wireless channels with multiple antennas both at the transmitter and receiver. The pioneer work of [1] [2] give the basic concept of MIMO antennas. By installing a high number of antennas at the transmitter and receiver, the capacity of MIMO would be enhanced compared with conventional communication systems such as single input single output (SISO), single input multiple output (SIMO), and Multiple input single output (MISO) [3] [4]. However, by increasing multiple antennas the signal correlation affects the channel capacity in partially and fully correlated environment[5].

In this paper,we examine the capacity of MIMO in the correlated environment by using scattering parameters. MIMO systems are very smart techniques in improving the capacity of wireless communication in the rich multipath atmosphere [6]. We obtained the scattering parameter matrix via Ansoft HFSS based antenna simulation. In fact, we only consider the case of $2 \times 2$ MIMO to explain in detail the effect of scattering parameters on the capacity of MIMO in correlated environment. We focused our analysis of capacity for partially and fully correlation situation. The capacity of MIMO based antenna systems givesa reasonable increase in capacity by installing a high number of antennas at the transmitter and receiver side. However, this method introduces not only signal correlation [7], but envelope correlation as well [8]. Reflection and transmission coefficient of scattering parameters are attained by designing microstrip patch antenna. The desired results to analyze the antenna performance are acquired by using a probe feed mechanism in patch antenna design.

The plots of MIMO for two transmit and receive antenna are examined. We exploited scattering matrix with the help of scattering parameters of reflection and transmission using suggested design. Consequently, the effects of partially and fully correlated MIMO system on capacity by using scattering matrix are studied.Comparative analysis of the percentage decrease of capacity in the presence of scattering matrix is also observed in Figure 5. The value against these Plots are shown in Table I. A detailed analysis proposed antenna and the expression of capacity in term of scattering parameters is presented in the next sections.

\section{Evaluation of MIMO Antennas}

The performance analysis of antenna such as Gain and Return Loss can be used to predict the behavior of MIMO based antenna array design. In [9], the author discuss that, correlation between the signals is a good measure to analyze a behavior of MIMO antenna system. The expression, which gives the correlation of complex signals, is referred as signal correlation [9]-[14]. The equation which gives the correlation co-efficient in case of MIMO antennas is dependent on the scattering parameters of reflection and transmission co-efficient which give birth to scattering matrix. This scattering matrix in term of correlation co-efficient $\rho$ can be expressed as [13][14]. 
$\rho_{s}=\frac{-\left(S_{11}^{*} S_{12}+S_{21}^{*} S_{22}\right)}{\sqrt{1-\left|S_{11}\right|^{2}-\left|S_{21}\right|^{2} 1-\left|S_{22}\right|^{2}-\left|S_{21}\right|^{2}}}$

It can easily be noticed from Equation 1 that, the reflection and transmission co-efficient affect the performance of antenna in term of system capacity. This particular phenomenon of descent in capacity is discussed in this article.

\section{MIMO Antenna Design}

Consider a two element parallel placed patch antenna with respect to y-axis as shown in Figure 1. This antenna is designed for $2.6 \mathrm{GHz}$ MIMO applications. This MIMO system is configured by using a probe feed mechanism in such a way that patch element 1 and 2 are placed parallel to each other. With the aid of Ansoft HFSS, length, width and height of each patch antenna is set at $60 \mathrm{~mm}, 20 \mathrm{~mm}$ and $12 \mathrm{~mm}$ respectively. Each patch is excited by using $1.2 \mathrm{~mm}$ thick $50 \Omega$ probes. In this case, the ground planeis making conducted by deploying $0.5 \mathrm{~mm}$ thick copper sheet having dimension $120 \times 90 \mathrm{~mm}^{2}$. The respective plots of gain and return loss are discussed in the next section.

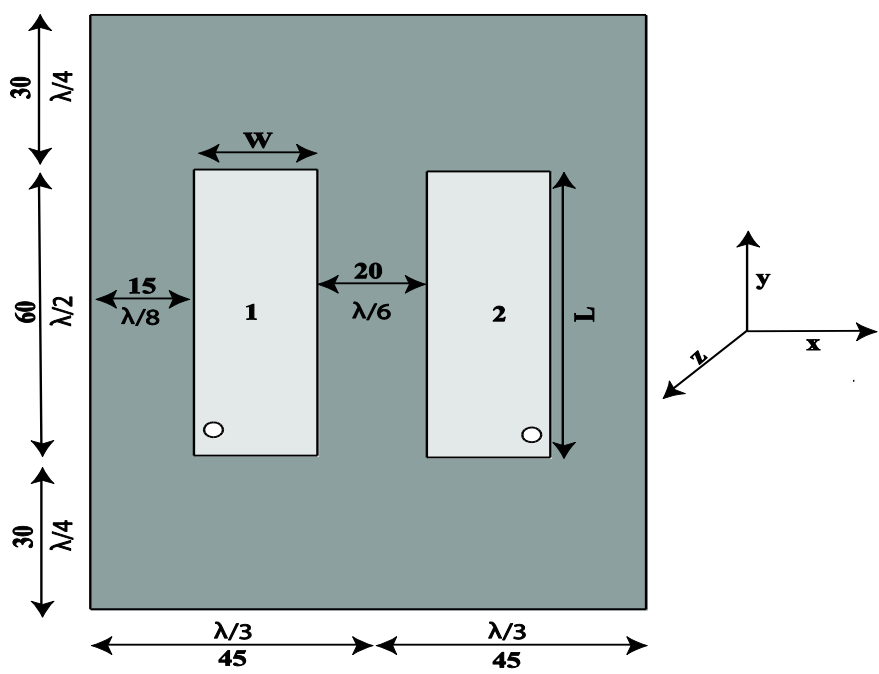

Figure 1: Patch antenna array design of MIMO system antenna array.

Simulated Results and Discussion of HFSS Based Design Antenna

Consider a two element parallel placed patch antenna with respect to y-axis as shown in Figure 2. This antenna is designed for 2.6 GHz for MIMO applications. Return loss of $-25 \mathrm{~dB}$ and $-26 \mathrm{~dB}$ can easily be noticed for these parallel placed patch antenna elements 1 and 2 . The gain of these patch antenna elements 1 and 2 with respect to isotropic antenna $7 \mathrm{dBi}$ which is presented in this simulated work as depicted in Figure $3 \mathrm{At}-150^{\circ}$ and $150^{\circ}$, the null point can easily be observed for phi $0^{\circ}$ and $45^{\circ}$ and $90^{\circ}$. Another important factor is the main lobe, which is observed at $0^{0}$ as shown in Figure 3. Moreover, Half Power Beamwidth (HPBW) of $4 \mathrm{dBi}$ is also cleared in Figure 3.

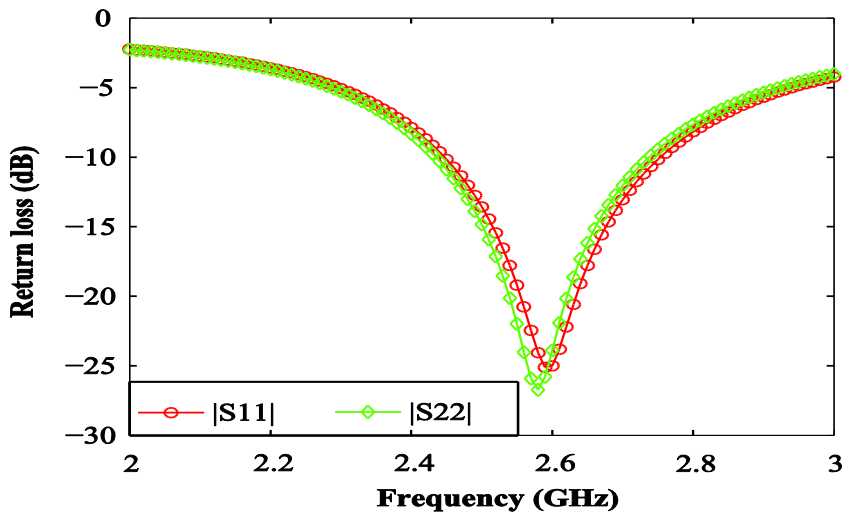


Figure 2: Simulated return loss of MIMO system antenna array.

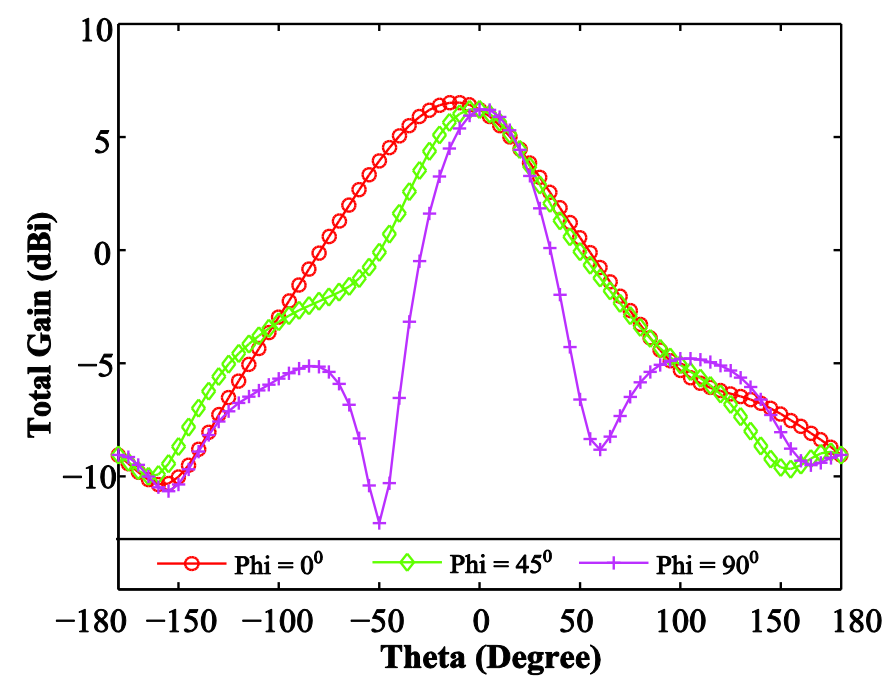

Figure 3: Simulated radiation pattern of MIMO system antenna array.

\section{Simulated Results and Discussion of Capacity Plots in the Presence of Scattering Parameters}

A brief description of MIMO capacity in the presence of scattering parameters is discussed. In fact, we only discussed $2 \times 2$ transmit and received MIMO antennas, as shown in Figure 4, less than $10 \mathrm{bps} / \mathrm{Hz}$ of capacity is seen at and SNR of 20dB. This value goes down gradually as we move from right to left in the value of SNR. Rest of the cases, pictured in Figure4, show a relative decrease with respect to i. i. d. in capacity observed from $-10 \mathrm{~dB}$ to $20 \mathrm{~dB}$. The descent of capacity is the effect of scattering matrix while using Equation 5. Moreover, at transmitter and receiver, the correlation matrices are defined as[15].

$\mathbf{R}^{T}=\mathbf{R}^{1 / 2}\left(\mathbf{R}^{1 / 2}\right)^{\mathbf{H}}$,

$\mathbf{R}^{\mathbf{R}}=\mathbf{R}^{\mathbf{1 / 2}}\left(\mathbf{R}^{\mathbf{1 / 2}}\right)^{\mathbf{H}}$,

Where ' $R$ ' is the receive correlation matrix at transmitter and receiver. On the other hand the capacity equation for independent identically distribute channel matrix ' $\mathrm{H}$ ' is discussed in[16]

$C=\log _{2}\left[\operatorname{det}\left(I_{N r}+\frac{P_{t}}{N_{0} N_{t}} H H^{H}\right)\right] \operatorname{bps} / H z$.

In addition, in the presence of scattering parameters the modified form of capacity equation is elaborated in[17]-[19]

$C=\log _{2}\left[\operatorname{det}\left(I_{N r}+\frac{P_{t}}{N_{0} N_{t}} \varphi_{r} H H^{H}\right)\right] \operatorname{bps} / \mathrm{Hz}$,

where $\boldsymbol{I}_{\boldsymbol{N r}}$ is the identity matrix of number of received antenna as. $\boldsymbol{P}_{\boldsymbol{t}}$ is transmit power and $\boldsymbol{H}$ is the channel matrix, $\boldsymbol{H}^{\boldsymbol{H}}$ is the conjugate transpose of channel matrx, $\boldsymbol{\varphi}_{r}$ is the received correlation matrix in term of scattering parameters and defined as[19]

$\boldsymbol{\varphi}_{r}=\left(\begin{array}{cc}\mathbf{1} & \boldsymbol{\rho}_{s} \\ \boldsymbol{\rho}_{s}^{*} & \mathbf{1}\end{array}\right)$,

where $\boldsymbol{\rho}_{s}$ is shown in Equation(). It is valid only when perfect channel state information is available at receiver [20].The percentage decrease in capacity with respect to scattering matrix for $2 \times 2$ MIMO is depicted in Figure 5 . These plots shown pointing to point descent in capacity with respect to SNR value in $\mathrm{dB}$. For $2 \times 2$ MIMO antenna system less than 80 percentage decrease in capacity is cleared in case of partially and fully correlated environment. This value goes down and reaches 30,20 and 10 percent in capacity, by looking in the value of SNR at $20 \mathrm{~dB}$. The values against this decrease are shown in Table(I). 


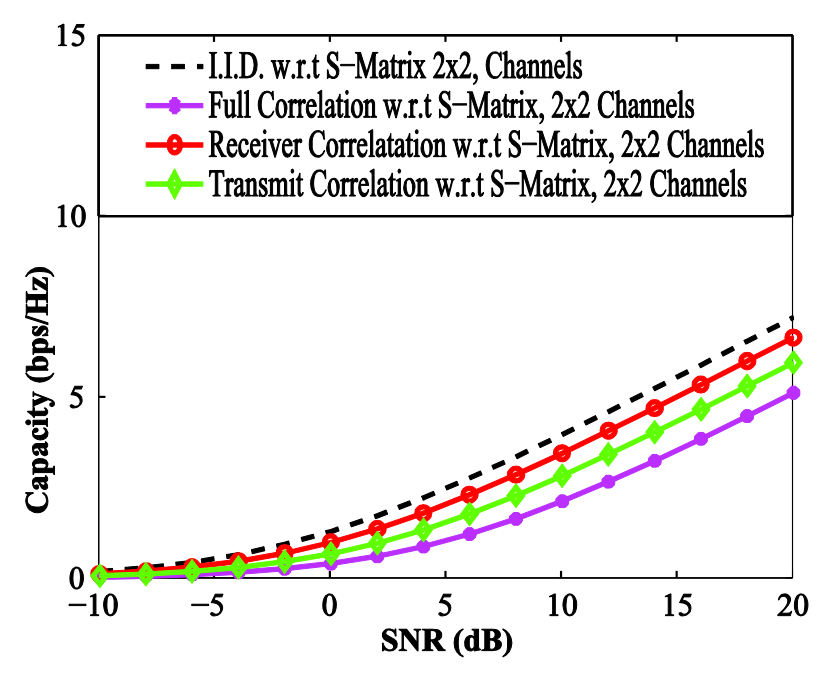

Figure 4:Comparision of i.i.d. and correlated $2 \times 2$ MIMO channel in the presence of scattering parameters.

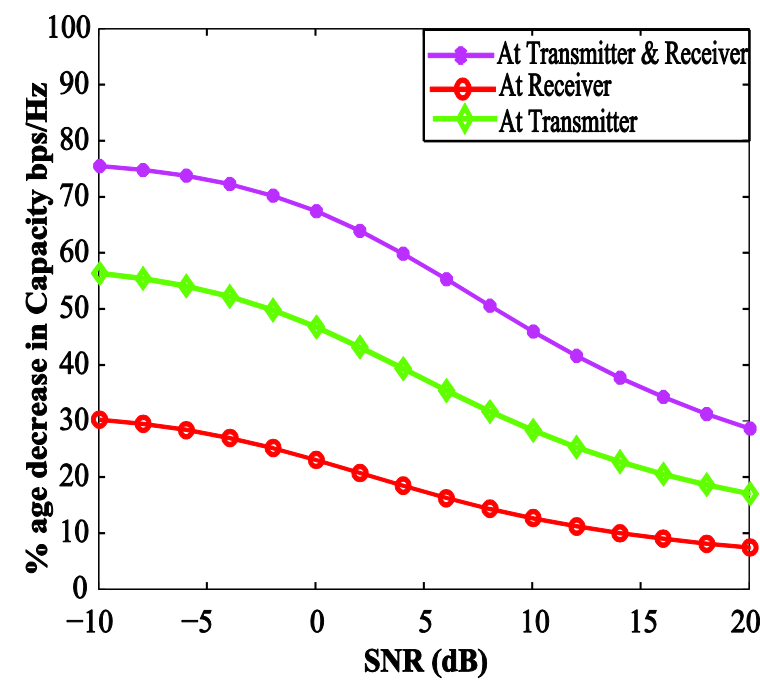

Figure 5: $\%$ age reduction in capacity from i.i.d. for $2 \times 2$ MIMO channel.

\section{Conclusions}

In this paper, we exposed the analysis of correlated MIMO channels using scattering parameters. The scattering matrix is extracted using scattering parameters obtained from proposed MIMO antenna design. Then the comparative analysis of $2 \times 2$ MIMO system is discussed. In fact, we elaborated the capacity of the MIMO antenna array in partially and fully correlated atmosphere. This environment of capacity is compared with i.i.d. in the presence of scattering matrix for the above mentioned cases. These observations are shown in Figure 4. The decline in percentage is also elaborated in Figure 5. The value against these descents of percentage is shown in Table I.

Table I $\%$ age decrease in capacity for $\mathrm{N}_{\mathrm{t}}=\mathrm{Nr}=2$ in the presence of scattering parameters

\section{References}

[1] G. J. Foschini, "Layered space-time architecture for wireless communication in a fading environment when using a multi - element antenna”, Bell Labs Technical Journal, vol. 10, pp 41-59, 1996.

[2] S. M. Alamouti, “A simple transmit diversity technique for wireless communications", IEEE Journal on Selected Areas in Communications, vol. 16, pp. 1451-1458, 1998.

[3] A. F. Molisch, "Wireless Communications". West Sussex, England: John Wiley and Sons, 2005.

[4] S. Haykin, M. Moher, "Modren Wireless Comunications", Pearson Education, 2005.

[5] Y. S. Cho, J. Kim, W. Y. Yang, and C. G. Kang, “MIMO-OFDM Wireless Communication with MATLAB'”. John Wiley and Sons, 2010 .

[6] R. Janaswamy, " Effect of element mutual coupling ont the capacity of fixed length linear array", IEEE Antenna and Wireless Propagation Letter, Vol. 1, Pg. 157-160, 2002.

[7] W. F. Tsen and H. Li, “ IEEE Antenna and Wireless Propagation Letter, Vol. 8, pp 1295-1298, 2009.

[8] Y. Fan, Y. Fei, B. K. Lau, and J. S. Thompson. "Optimal single-port matching impedance for capacity maximization in compact MIMO arrays". IEEE Transaction on Antenna and Propagation, vol. 56(no. 11): pp 3566-3575, 2008.

[9] C. A. Balanis. Antenna Theory and Design. John Wiley \& Sons, 2003.

[10] P. S. Kildal and K. Rosengren. "Correlation and capacity of MIMO systemsand mutual coupling, radiation efficiency, and diversity gain of their antennas:Simulation and measurements in reverberation chamber and anechoicchamber”. IEEE Communication Magazine, vol. 12(no. 12):pages 102-112,2004.

[11] J. W. Wallance and M. A. Jensen. "Mutual coupling in MIMO WirelessSystem a rigorous network theory analysis". IEEE Transaction on WirelessCommunication, vol. 3(no. 4):pages 1317-1325, 2004.

[12] T. Svantesson. "Correlation and Channel Capacity of MIMO system employingmultimode antennas". IEEE Transaction on Vehicular Technology, vol. 51(no. 6):pages 2440-2447, 2007.

[13] S. Lu, H. T. Hui, and M. Bialkowski. "Optimizing MIMO channel capacities under the influence of antenna mutual coupling". IEEE Antenna WirelessPropagation Letter, vol. 7:pages 287-290, 2008.

[14] X. Chen, P. S. Kildal, J. Carlsson, and J. Yang. "Comparison of ergodic capacitiesfrom wideband MIMO antenna measurements in reverberation chamberand anechoic chamber”. IEEE Antenna and Wireless Propagation Letter,vol. 10:pages 446-449, 2011. 
[15] Z. N. Chen, X. N. Low, and S. P. Terence. "Analysis and Optimization ofCompact Suspended Plate MIMO Antennas". IEEE Transaction on Antennaand Propagation, vol. 59(no. 1):pages 263-269, 2011.

[16] Ada S. Y. Poon, R. W. Brodersen, and D. N. C. Tse. "The Signal Dimensionsin Multiple-Antenna Channels". Global Telecommunications Conference, vol.3:pages 1247-1251, 2003.

[17] J. Thaysen and K. B. Jakobsen. "Envelope Correlation in (N,N) MIMOantenna arrray from scattering parameters". Micro. Opt. Tech. Letter, vol.48(no. 5):pages 832-834, 2006

[18] M. K. Ozdemir, E. Arvas, and H. Arslan. "Dynamics of spatial correlationand implications on MIMO systems". IEEE Communication Magazine, vol.42(no. 6):pages S14-S19, 2004.

[19] S. Dossche, S. Blanch, and J. Romeu. "Optimum antenna matching to minimize signal correlation on a two-port antenna diversity system". ElectronicLetter, vol. 40(no. 19):pages 1162-1165, 2004.

[20] J. Zhao and J. Wang. "Correlation reduction in antennas with metamaterialbased on newly designed SRRs ". Asia-Paci_c Symposium on Electromagneticand Compatibility, 12-10:pages 981-984, April 2010. 